\title{
Adhesive-bandage-type Blood-leakage Sensor Fabricated by Screen-offset Printing
}

\author{
Ken-ichi Nomura, ${ }^{*}$ Yoshinori Horii, and Hirobumi Ushijima \\ Flexible Electronics Research Center, National Institute of Advanced Industrial Science and Technology, \\ 1-1-1 Higashi, Tsukuba, Ibaraki 305-8565, Japan
}

(Received October 4, 2018; accepted January 30, 2019)

Keywords: printed electronics, screen-offset printing, adhesive, blood leakage, dielectric relaxation

We developed a new blood-leakage sensor for hemodialysis therapy. In this sensor, medical gauze is arranged on medical adhesive tape, which contains detection electrodes formed by screen-offset printing. This sensor can be used similarly to an adhesive bandage. We proved that the sensor can distinguish between blood and phosphate-buffered saline (substitute for sweat) on the basis of the dielectric relaxation property of blood. The limit of detection of the sensor is as low as $6 \mu \mathrm{L}$, and hence, its performance is considerably better than that of other sensors reported previously.

\section{Introduction}

Hemodialysis is a very important treatment procedure for patients needing renal replacement therapy. In this procedure, two needles are inserted into the patient's blood vessels to draw and return blood, and the blood is cleaned using external equipment. During the procedure, blood leakage may sometimes occur because of the accidental removal of the needle. The patients themselves may not notice this dangerous situation because they may be staying motionless, may not be paying any attention, or may be sleeping during hemodialysis therapy, which takes 4-8 h. Medical doctors and nurses may also find it difficult to notice bleeding because it may not be feasible for them to continuously monitor blood leakage for the entire duration of the therapy session. Therefore, the development of a blood-leakage sensor is very important to provide safe and secure hemodialysis therapy, and accordingly, many researchers have attempted to develop such a sensor. ${ }^{(1-4)}$

Patients suffering from renal failure require hemodialysis therapy two or three times a week, and the number of such patients is considered to be more than 300000 in Japan ${ }^{(5)}$ and at least 3 million globally. ${ }^{(6)}$ Hence, the blood-leakage sensor should be amenable to mass production and disposable. Therefore, the cost of the sensor should be low. Another important consideration is that patients should not feel uncomfortable when the sensor is attached to the body. For example, a dementia patient may try to remove the sensor unconsciously if the sensor is prominent and causes a feeling of strangeness. Previously, we developed a blood-leakage

*Corresponding author: e-mail: k-nomura@aist.go.jp

https://doi.org/10.18494/SAM.2019.2141 
sensor with detection electrodes fabricated on textiles; ${ }^{(7)}$ however, such a sensor must ultimately be fixed to the body using adhesive tape.

To address these issues, we developed a new adhesive-bandage-type blood-leakage sensor that can easily be used merely by sticking it on the patient's body. This sensor is composed of adhesive tape with printed interdigitated electrodes to detect blood and medical gauze to blot blood. In this report, we explain the technique used for fabricating the electrodes on the adhesive tape. In addition, we elucidate the mechanism of detecting blood using the electrodes and the detection ability of the sensor.

\section{Experimental Procedure}

\subsection{Fabrication of sensor electrodes on adhesive materials}

As mentioned above, a large number of patients require hemodialysis therapy and hence the blood-leakage sensors used for these patients should be disposable. Therefore, these sensors must be amenable to mass production at low cost. Accordingly, printing using conductive ink is suitable for the fabrication of the sensor electrodes. Many printing techniques exist, such as flexography, gravure printing, offset printing, and screen printing. However, it is considerably difficult to form patterns on adhesive materials using these conventional printing techniques because masks and adhesives stick together during printing. In addition, inkjet printing, as a form of maskless printing, is not appropriate for mass production because it takes considerably longer to print patterns than other printing techniques.

To overcome these problems, we applied a screen-offset printing method ${ }^{(7-13)}$ that we previously developed to fabricate patterns on an adhesive material. Figure 1 shows a schematic image of the screen-offset printing method. In this method, patterns of conductive ink are formed on a blanket made of polydimethylsiloxane (PDMS) by the screen-printing technique, and then, the patterns on the PDMS blanket are transferred to a substrate. During the transfer process, the substrate can be easily peeled off from the blanket even though the substrate is made of a sticky material. As a result, the conductive ink can be transcribed onto the adhesive to form the electrode patterns for the sensor. ${ }^{(11)}$ In the present experiment, we first screen-

Screen printing on PDMS blanket

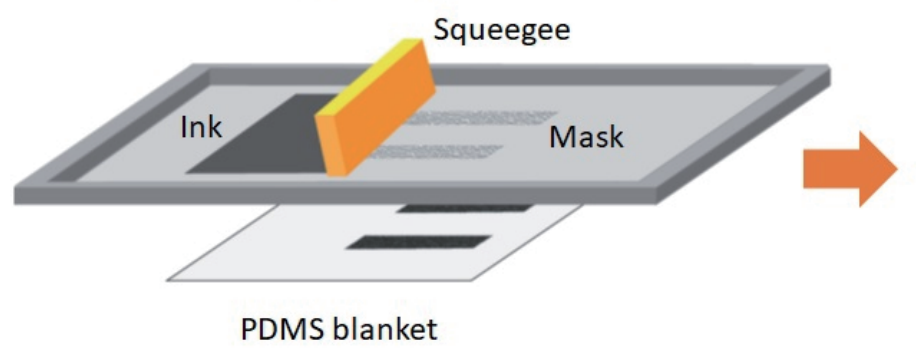

Pattern transfer to final substrate

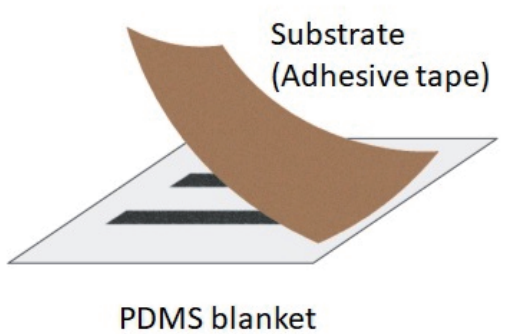

Fig. 1. (Color online) Schematic of screen-offset printing. 
printed an interdigitated pattern using Ag ink (SSP2801, Toyobo) on the blanket using a screenprinting machine (Cube1515, Mino Group). Then, we thermally annealed the ink on the blanket at $150{ }^{\circ} \mathrm{C}$ for $30 \mathrm{~min}$. Subsequently, we transferred the ink patterns to medical adhesive tape [Fig. 2(a)]. Note that we overprinted (double-printed) the patterns on the blanket to obtain the maximum thickness for the electrodes and thereby lower the risk of wire disconnection. We arranged two-ply medical gauze on top of the tape with the Ag-ink patterns to obtain the adhesive-bandage-type sensor [Fig. 2(b)]. Figure 2(c) shows the sensor on which $5 \mu \mathrm{L}$ of human whole blood (Tennessee Blood Service purchased via Bizcom Japan) was dropped. The sensor appears similar to the widely used adhesive bandages; this should contribute to decreasing the psychological discomfort of patients.

\subsection{Electrical measurement}

The electrical properties of the fabricated adhesive-bandage-type sensor were measured using an inductance-capacitance-resistance meter (3532-50 LCR HiTESTER, Hioki E.E.). In this experiment, the impedance $Z$ and phase $\theta$ were measured at frequencies $f$ of $1 \mathrm{kHz}-5 \mathrm{MHz}$. From the results, the lumped capacitive components $C$ in a simple resistor-inductor-capacitor model were estimated using

$$
C=-\frac{1}{2 \pi f Z \sin \theta} .(\mathrm{F})
$$

Here, the inductive components were assumed to be zero. In the frequency range of $1 \mathrm{kHz}-5$ $\mathrm{MHz}$, data were collected at 200 measurement points. At each measurement point, the data were obtained twice and the average value was considered.

\section{Results and Discussion}

First, we checked whether the dielectric relaxation of blood is properly detected by our developed sensor. Figure 3 shows the results for the capacitance as a function of frequency

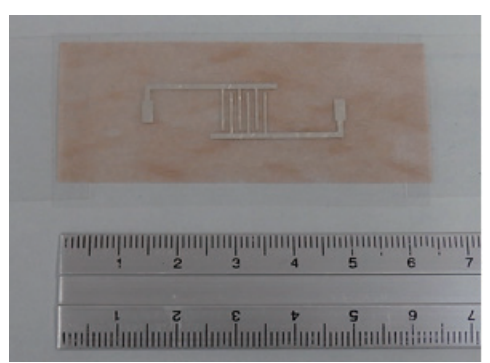

(a)

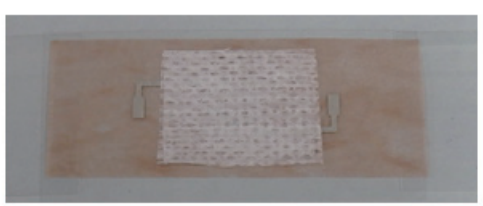

(b)

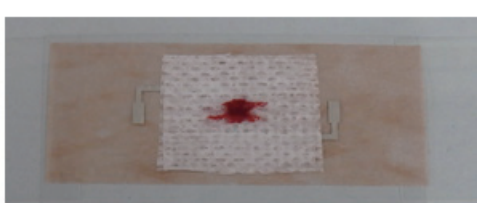

(c)

Fig. 2. (Color online) Images of adhesive-bandage-type sensor (a) before and (b) after attaching gauze. (c) Photograph of sensor after $5 \mu \mathrm{L}$ of blood was dropped on it. 


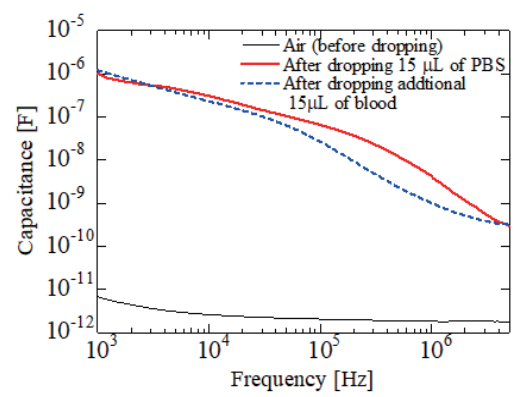

Fig. 3. (Color online) Capacitance as a function of frequency of sensor obtained before dropping PBS, after dropping $15 \mu \mathrm{L}$ of PBS, and after dropping additional $15 \mu \mathrm{L}$ of blood.

obtained before dropping phosphate-buffered saline (PBS), after dropping $15 \mu \mathrm{L}$ of PBS, and after dropping an additional $15 \mu \mathrm{L}$ of blood. The slope of the spectrum obtained after dropping PBS is different from that obtained after dropping the additional blood. In particular, in the frequency range of $50-300 \mathrm{kHz}$, the spectral slope for blood is substantially different from that for PBS. This substantial difference seems to be due to the dielectric relaxation of blood because it has been reported that blood exhibits relaxation in this frequency range. ${ }^{(14)}$ In addition, this result indicates that the detection of the leaked liquid as blood is correct.

Next, we elucidated the limit of detection (LOD) of the developed sensor using the scheme previously reported in Ref. 7. First, we measured the spectra when blood or PBS (up to $50 \mu \mathrm{L}$ ) was dropped onto the sensor. In addition, we obtained the spectra for the sensor on which 50 $\mu \mathrm{L}$ of PBS was dropped and left to dry at room temperature, because if blood leakage occurs during hemodialysis, blood continuously leaks, whereas sweat may dry. Then, we selected a threshold value at a certain frequency $f_{1}$; this selection eliminated the unstable condition under which less blood or PBS is dropped onto the sensor. Next, we selected two other frequencies $\left(f_{2}\right.$ and $\left.f_{3} ; f_{2}<f_{3}\right)$ and obtained capacitances at these frequencies referred to as $C_{2}$ and $C_{3}$, respectively. Finally, the subtracted values obtained from the two points, namely, $\log _{10} C_{2}-$ $\log _{10} C_{3}$, were compared to determine whether blood had leaked onto the sensor.

Figures 4(a) and 4(b) show the representative data for the frequency dependences of the capacitance when blood and PBS were dropped, respectively. Figure 4(c) shows the spectra for the sensor on which $50 \mu \mathrm{L}$ of PBS was dropped and left to dry. Note that the spectrum for $50 \mu \mathrm{L}$ of PBS shown in Fig. 4(b) is the same as that for 0 min shown in Fig. 4(c). From these measurement results, the values of $C_{1}, C_{2}$, and $C_{3}$ at $f_{1}, f_{2}$, and $f_{3}$, respectively, are extracted. In this experiment, we chose 10.1, 51.3, and $202 \mathrm{kHz}$ at $f_{1}, f_{2}$, and $f_{3}$, respectively, which are the same frequencies as those used in a previous study. ${ }^{(7)}$ Figure 5 shows the capacitance at 10.1 $\mathrm{kHz}\left(C_{1}\right)$ as a function of the total volume of (a) blood or (b) PBS dropped and the capacitance as a function of the drying time of the sensor on which PBS was dropped (c). Furthermore, Fig. 6 shows the values of $\log _{10} C_{2}-\log _{10} C_{3}$ for each condition. For example, it is assumed that 50 


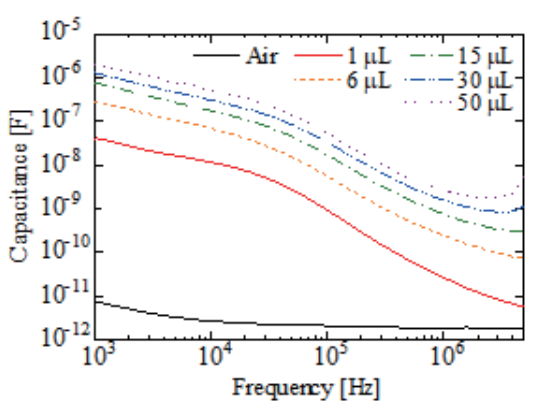

(a) Blood

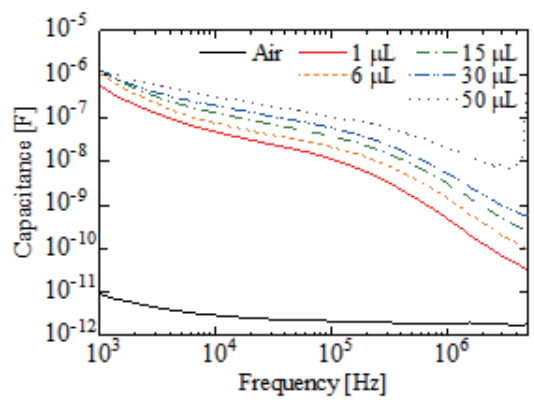

(b) PBS

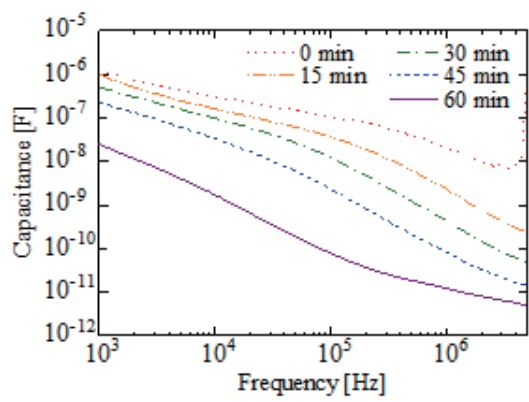

(c) PBS (drying)

Fig. 4. (Color online) Capacitance as a function of frequency obtained when (a) blood or (b) PBS with a volume of up to $50 \mu \mathrm{L}$ was dropped. (c) Spectra for sensor on which $50 \mu \mathrm{L}$ of PBS was dropped and left to dry.

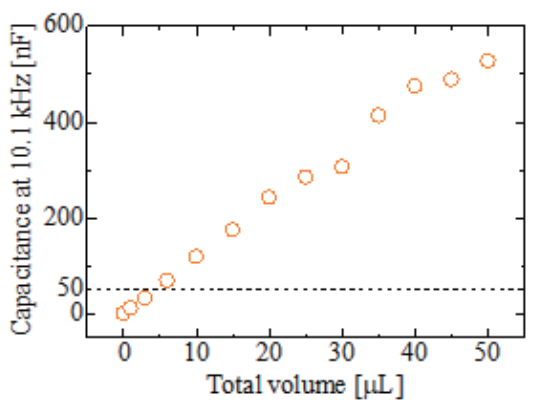

(a) Blood

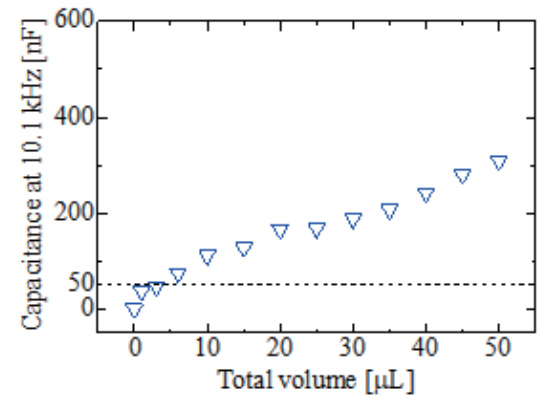

(b) PBS

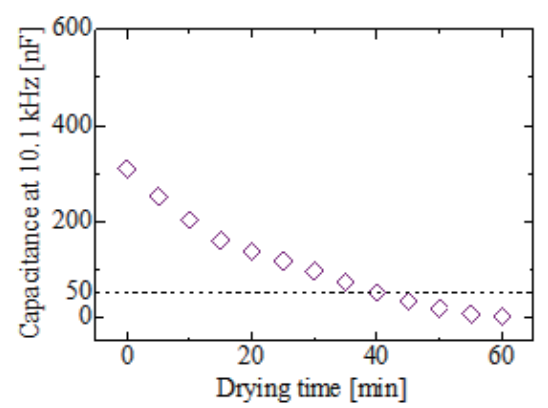

(c) PBS (drying)

Fig. 5. (Color online) Capacitance at $10.1 \mathrm{kHz}$ as a function of volume of (a) blood or (b) PBS dropped onto sensor. (c) Spectra for sensor on which $50 \mu \mathrm{L}$ of PBS was dropped and left to dry. The assumed threshold value $(50 \mathrm{nF})$ is indicated for reference in each figure. 


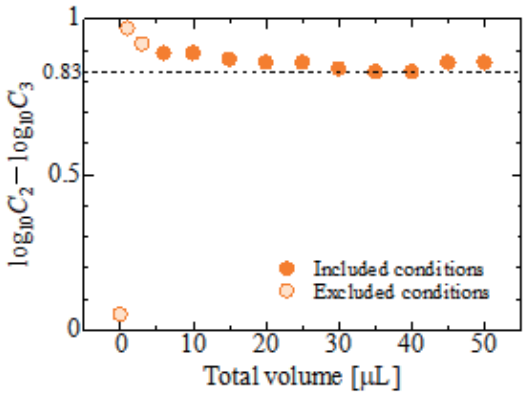

(a) Blood

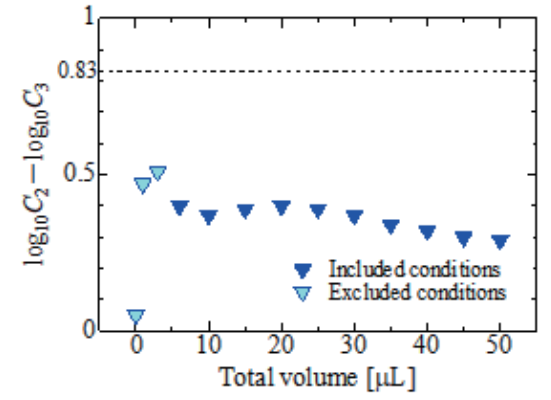

(b) PBS

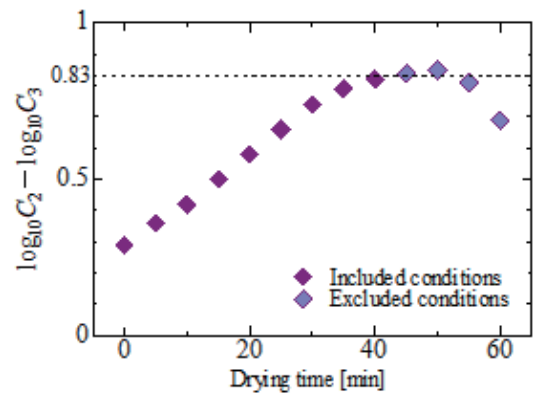

(c) PBS (drying)

Fig. 6. (Color online) $\log _{10} C_{2}-\log _{10} C_{3}$ as a function of total volume of (a) blood and (b) PBS dropped . (c) Dryingtime dependence of values for sensor on which $50 \mu \mathrm{L}$ of PBS was dropped. The minimum value for blood (i.e., 0.83) is indicated in all the figures for reference.

$\mathrm{nF}$ is the threshold value of $C_{1}$; the conditions below this value are excluded. In this case, we remove the conditions of $0-3 \mu \mathrm{L}, 0-3 \mu \mathrm{L}$, and 45-60 min in Figs. 5(a)-5(c), respectively, and show the included and excluded conditions in Fig. 6. All the included values of $\log _{10} C_{2}-\log _{10} C_{3}$ shown in Fig. 6(a) are higher than those shown in Figs. 6(b) and 6(c). These results indicate that the sensor properly recognizes blood. In addition, the LOD of the sensor is found to be $6 \mu \mathrm{L}$, which is lower than that of other recently reported sensors $\left(10^{(1)}\right.$ and $\left.15 \mu \mathrm{L}^{(7)}\right)$.

Next, we address the question why the detection ability of the sensor developed in this study is higher than that reported in Ref. 7. Figures 7(a) and 7(b) show schematic images of the cross sections of the sensors reported in Ref. 7 and employed in this study, respectively. The sensor electrodes in Fig. 7(a) were formed on the cotton textile and were exposed to air directly, whereas those of the bandage-type sensor were sandwiched between medical tape and medical gauze [Fig. 7(b)]. We first changed the cloth of the bandage-type sensor from medical gauze to cotton textile [Fig. 7(c)], to facilitate the comparison with the case shown in Fig. 7(a) by employing the same cloth, and the LOD value was estimated as described above. Figure 8 shows the capacitance of the bandage-type cotton-textile sensor at $10.1 \mathrm{kHz}\left(C_{1}\right)$ as a function of the total volume of (a) blood or (b) PBS dropped (up to $50 \mu \mathrm{L}$ ) and (c) the capacitance as a function of the drying time of the sensor on which $50 \mu \mathrm{L}$ of PBS was dropped. Also, Fig. 9 shows the frequency dependence of $\log _{10} C_{2}-\log _{10} C_{3}$ for each condition, where $C_{2}$ and $C_{3}$ are the capacitance values at 51.3 and $202 \mathrm{kHz}$, respectively. When the threshold value in Fig. 8 is 


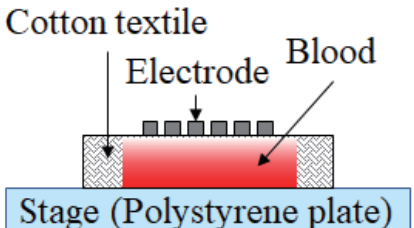

Stage (Polystyrene plate)

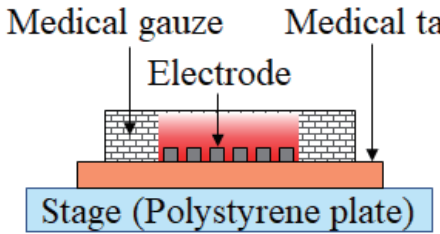

$\underline{6 \mu \mathrm{L}}$

(b)

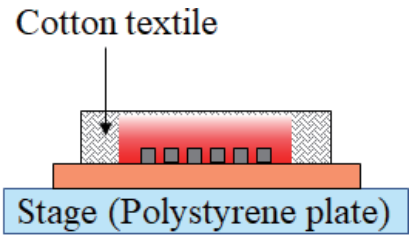

$\underline{3 \mu \mathrm{L}}$

(c)

Fig. 7. (Color online) Schematic images of the cross sections of sensors (a) reported in Ref. 7 and (b) employed in this study. The sensor shown in (c) was used for additional experiments in which the cloth shown in (b) was changed from medical gauze to cotton textile.

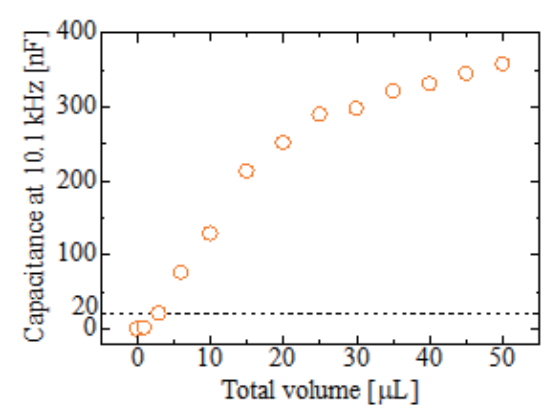

(a) Blood

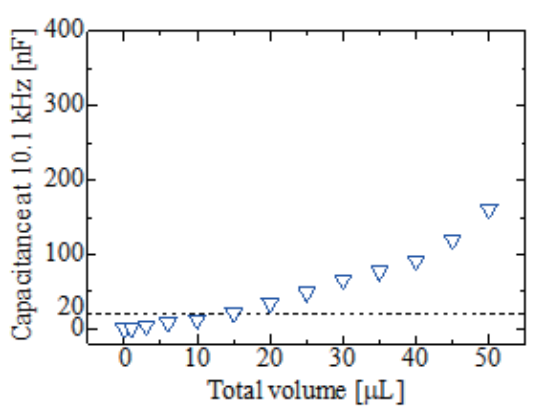

(b) PBS

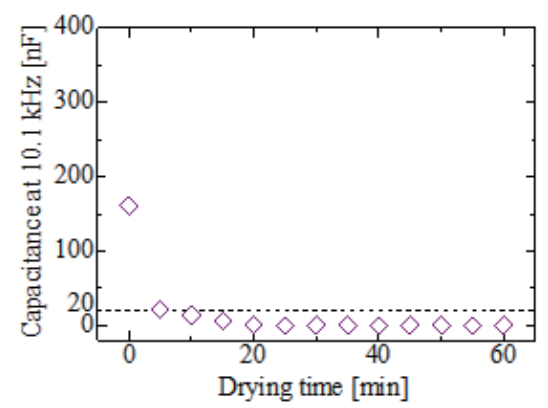

(c) PBS (drying)

Fig. 8. (Color online) Capacitance of bandage-type cotton-textile sensors at $10.1 \mathrm{kHz}$ as a function of volume of (a) blood or (b) PBS dropped onto sensor. (c) Spectra for sensor on which $50 \mu \mathrm{L}$ of PBS was dropped and left to dry. The assumed threshold value $(20 \mathrm{nF})$ is indicated for reference in each figure.

assumed to be $20 \mathrm{nF}$, the LOD of this bandage-type cotton-textile sensor became $3 \mu \mathrm{L}$, which is better than that corresponding to the case depicted in Fig. 7(a) $(15 \mu \mathrm{L})$. As reported in Ref. 7, it took 2-3 min to finish the electrical measurement scan, and blood gradually dried from the tops of the cloths during the measurements. This drying may prevent erythrocytes in blood from showing dielectric relaxation, which should degrade the sensor performance. However, 


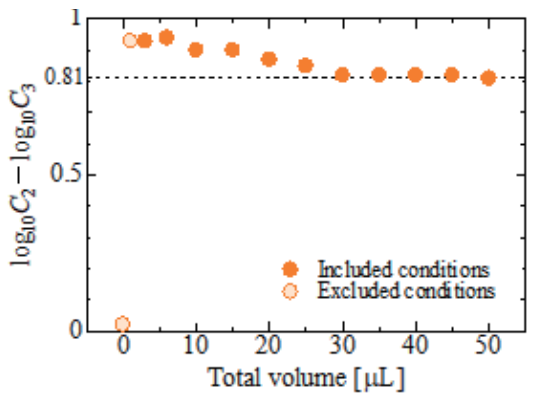

(a) Blood

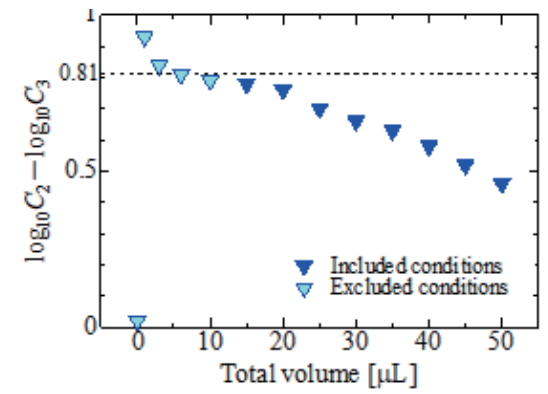

(b) PBS

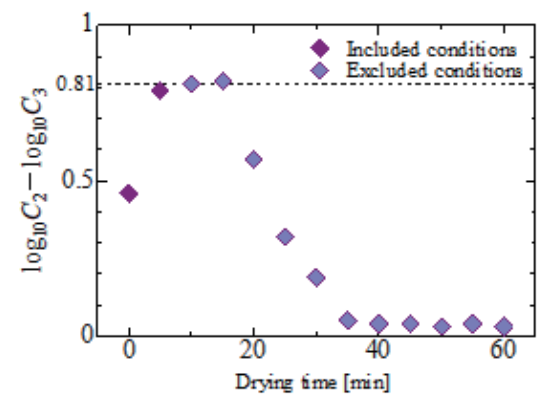

(c) PBS (drying)

Fig. 9. (Color online) $\log _{10} C_{2}-\log _{10} C_{3}$ of the bandage-type cotton-textile sensor as a function of total volume of (a) blood and (b) PBS dropped. (c) Drying-time dependence of values for sensor on which $50 \mu \mathrm{L}$ of PBS was dropped. The minimum value in the case of blood (i.e., 0.81) is indicated in all the figures for reference.

blood in the vicinity of the sandwiched electrodes shown in Figs. 7(b) and 7(c) should dry more slowly than the case shown in Fig. 7(a), which may contribute to the better LOD. Furthermore, the LOD of the adhesive-type cotton-textile sensor [Fig. 7(c)] is slightly lower than that of the medical-gauze sensor [Fig. 7(b)]. Figure 10 presents photographs of the cotton textile and the medical gauze with a size of $\sim 5 \times 5 \mathrm{~cm}^{2}$, on which $50 \mu \mathrm{L}$ of blood was dropped. It can be observed that the size of the blotted blood is larger in the cotton textile than in the medical gauze, which implies that when the same volume of blood is dropped, the electrode area covered by blood is larger in the cotton textile, especially when the volume of blood dropped is small. This characteristic seems to be the reason why the cotton textile showed slightly better performance than the medical gauze. However, medical gauze may be suitable for practical use because it is sterilized.

Finally, a considerable increase in capacitance is observed at $\sim 4 \mathrm{MHz}$ when $50 \mu \mathrm{L}$ of PBS is dropped. At around this frequency, the phase changes from minus to plus. This indicates that the sensor becomes predominantly inductive rather than capacitive. If this phase inversion occurs at frequencies lower than $1 \mathrm{MHz}$, it may cause inaccurate diagnosis. As the next step, we will improve the structure of the sensor so that such phase inversion can be suppressed. 


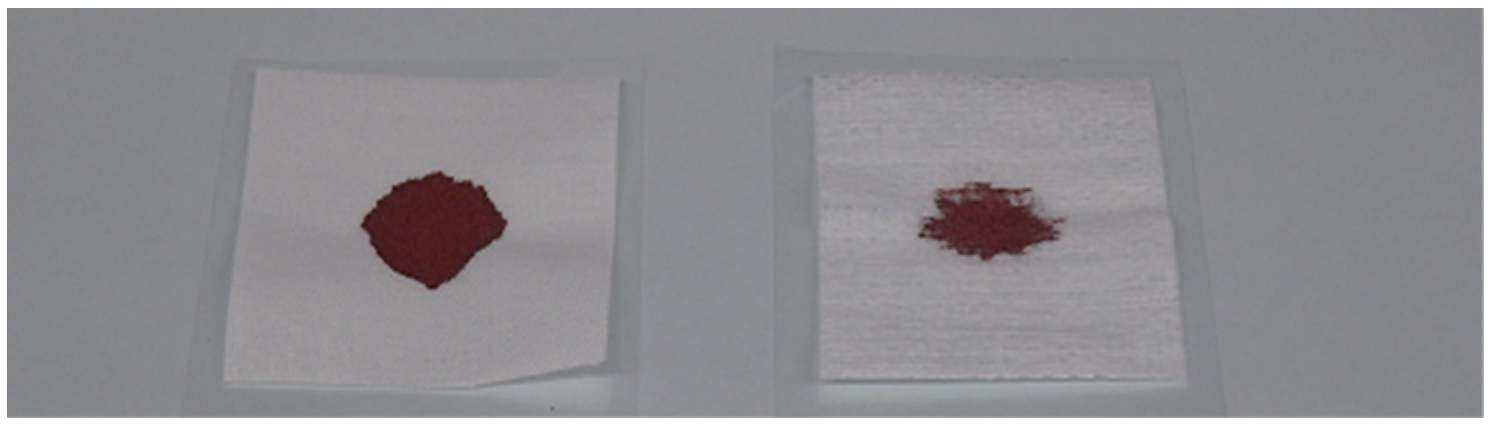

(a)

(b)

Fig. 10. (Color online) (a) Cotton textile and (b) medical gauze on which $50 \mu \mathrm{L}$ of blood was dropped.

\section{Conclusions}

We successfully demonstrated the simple fabrication of electrodes on adhesive tape using screen-offset printing and used this structure to develop an adhesive-bandage-type bloodleakage sensor. The printing-based fabrication technique is useful because it facilitates the mass production of this disposable sensor. In addition, the sensor developed can accurately distinguish between blood and PBS (substitute for sweat) on the basis of the dielectric relaxation property of blood. The sensor appears similar to the widely used adhesive bandages, which should contribute to decreasing the psychological discomfort of patients during hemodialysis therapy.

\section{References}

1 H.-C. Chuang, C.-Y. Shih, C.-H. Chou, J.-T. Huang, and C.-J. Wu: IEEE Sens. J. 15 (2015) 1515. https://doi. org/10.1109/JSEN.2014.2364302

2 Y.-C. Du, B.-Y. Lim, W.-S. Ciou, and M.-J. Wu: Sensors 16 (2016) 849. https://doi.org/10.3390/s16060849

3 C.-H. Lin, W.-L. Chen, C.-M. Li, M.-J. Wu, P.-T. Huang, and Y.-S. Chen: Healthcare Technol. Lett. 3 (2016) 290. https://doi.org/10.1049/ht1.2016.0051

4 P.-T. Huang, T.-L. Jong, C.-M. Li, W.-L. Chen, and C.-H. Lin: IEEE Trans. Biomed. Circuits Syst. 11 (2017) 784. https://doi.org/10.1109/TBCAS.2017.2695798

5 N. Miki: J. Jpn. Inst. Electron. Packag. 20 (2017) 109 (in Japanese). https://doi.org/10.5104/jiep.20.109

6 T. Liyanage, T. Ninomiya, V. Jha, B. Neal, H. M. Patrice, I. Okpechi, M. Zhao, J. Lv, A. X. Garg, J. Knight, A. Rodgers, M. Gallagher, S. Kotwal, A. Cass, and V. Perkovic: Lancet 385 (2015) 1975. https://doi.org/10.1016/ S0140-6736(14)61601-9

7 K. Nomura, Y. Horii, S. Kanazawa, Y. Kusaka, and H. Ushijima: Sensors 18 (2018) 240. https://doi. org $/ 10.3390 / \mathrm{s} 18010240$

8 K. Nomura, Y. Horii, H. Ushijima, H. Ikedo, and K. Nagase: Microelectron. Eng. 197 (2018) 23. https://doi. org/10.1016/j.mee.2018.05.002

9 S. Iwata, S. Otao, N. Imawaka, K. Yoshino, K. Nomura, Y. Horii, R. Kaji, H. Ushijima, and T. Isobe: J. Soc. Electr. Mater. Eng. 26 (2017) 78.

10 K. Nomura, R. Kaji, S. Iwata, S. Otao, N. Imawaka, K. Yoshino, R. Mitsui, J. Sato, S. Takahashi, S. Nakajima, and H. Ushijima: Sci. Rep. 6 (2016) 19947. https://doi.org/10.1038/srep19947

11 K. Nomura, H. Ushijima, K. Nagase, H. Ikedo, R. Mitsui, J. Sato, S. Takahashi, S. Nakajima, M. Arai, Y. Kurata, and S. Iwata: Jpn. J. Appl. Phys. 55 (2016) 03DD01. https://doi.org/10.7567/JJAP.55.03DD01

12 K. Nomura, Y. Kusaka, H. Ushijima, K. Nagase, H. Ikedo, R. Mitsui, S. Takahashi, S. Nakajima, and S. Iwata: J. Micromech. Microeng. 24 (2014) 095021. https://doi.org/10.1088/0960-1317/24/9/095021

13 K. Nomura, H. Ushijima, R. Mitsui, S. Takahashi, and S. Nakajima: Microelectron. Eng. 123 (2014) 58. https:// doi.org/10.1016/j.mee.2014.05.009

14 T. Chelidze: J. Non-Cryst. Solids 305 (2002) 285. https://doi.org/10.1016/S0022-3093(02)01101-8 